\title{
Bilateral Sertoli cell adenoma in gonads, ASSOCIATED WITH SEROUS CYSTADENOMA
}

\author{
Iván Fernandez-Vega ${ }^{1}$, Jorge Santos-Juanes ${ }^{2}$, Carmen García-Pravia ${ }^{2}$
}

${ }^{1}$ Service of Anatomic Pathology, University Hospital of Araba-Txagorritxu, Vitoria-Gasteiz, Spain

${ }^{2}$ Service of Anatomic Pathology, University Central Hospital of Asturias, Oviedo, Spain

\begin{abstract}
Complete androgen insensitivity syndrome is an extremely infrequent disease. The patients exhibit female phenotype because of insensitivity to the androgen receptor and may develop tumors, especially in their undescended gonads. We report a case of bilateral Sertoli cell adenoma in gonads with unilateral serous cystadenoma, in an elderly phenotypic woman with primary amenorrhea. We also provide radiological and pathological studies.
\end{abstract}

Key words: androgen insensitivity syndrome, gonadal cyst, Sertoli cell tumor.

We performed a comprehensive study of an elderly woman with primary amenorrhea complaining about respiratory problems. Almost always cancer disease can explain apparently unrelated medical signs.

A 78-year-old phenotypic woman with marked obesity came to our hospital suffering from orthopnea and irritative cough during the last year. Her past medical history revealed primary amenorrhea. Physical examination showed normal development of the breast, scarce axillary hair and a noteworthy palpable mass in her abdomen. Moreover, gynecological examination revealed that the vulva and perineum appeared normal but the vagina was short in length and the proximal part ended blindly, with an invisible cervix. Then, a computed tomography (CT) body scan was performed, and the images revealed a large solid abdominal mass of $20 \mathrm{~cm} \times 19 \mathrm{~cm}$ in size in the left adnexal region and a solid-cystic abdominal mass in the right adnexal region. The solid part of the right tumor was $4.5 \mathrm{~cm} \times 4 \mathrm{~cm}$ in size and the cystic part of the tumor was $8.5 \mathrm{~cm} \times 6 \mathrm{~cm}$ (Fig. 1A). Other CT scan findings were absent uterus and short and blind end vagina. Serum tumor markers were negative. The patient refused a cytogenetic study and any further investigations about her disease.

Due to suspected Morris syndrome, laparotomy was performed. Macroscopically the left side abdom- inal mass weighed $2.7 \mathrm{~kg}$. Both masses were completely encapsulated and exhibited a pale-yellow cut surface with other pink-white areas. The cystic mass had a smooth brownish surface and it was attached to the solid part (Fig. 1B).

Microscopically both solid lesions had a uniform tubular pattern with polygonal cells with granular and clear cytoplasm without conspicuous nucleoli. Cystic structure consisted of a fibrous wall lined by ciliated columnar epithelium (Fig. 1C). Peritumoral blood vessels showed a prominent layer of smooth muscle. The immunophenotype of the lesions was typical with strong and diffuse immunoreactivity for $\alpha$-inhibin (Fig. 1D) WT-1 and patchy moderate staining for calretinin (Fig. 1E). The rate of proliferation measured by Ki-67 was low. Immunohistochemical staining for estrogen receptors, progesterone receptors and Melan A were negative. We also noted remains of testicular structures with smooth muscle hyperplasia close to both tumors.

The pathological diagnosis was a classic variant of a bilateral well-differentiated Sertoli cell adenoma placed in both undescended testes with a serous cyst on the right side. Furthermore, the diagnosis of complete androgen insensitivity syndrome (CAIS) was suggested with the integration of clinical, radiolog- 

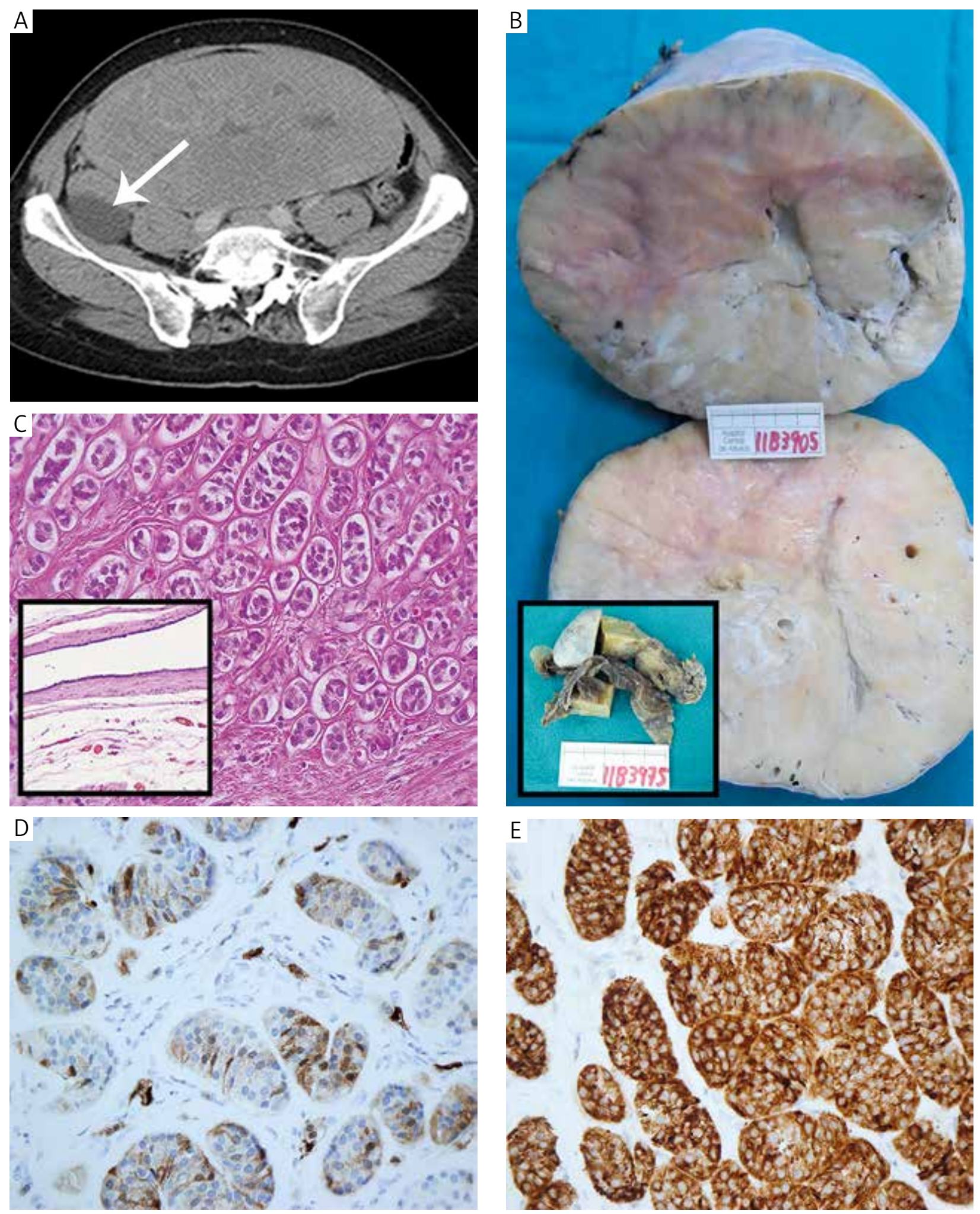

Fig. 1. Different imaging studies performed on the abdominal mass. A) CT scan shows the bilateral solid tumor with a cystic part on the right side (arrowed). B) Gross features of the tumor. The inset shows some pieces of the right-sided tumor with some fragments of the cystic part. C) Microscopic image of typical tubules from a well-differentiated Sertoli cell tumor. The inset displays a monostratified epithelium composed of columnar cells lining the cystic cavity (HE staining). D) Immunohistochemistry for inhibin shows strong cytoplasmic positivity in tumoral cells. E) The tumor cells display irregular and weak to moderate staining for calretinin. All immunostaining was performed by immunoperoxidase technique on paraffin-embedded tissue sections 
ical and pathological findings [1]. The patient is still being followed up regularly and she is healthy.

Although the risk of developing tumors in women with CAIS increases with age, Sertoli cell adenomas are not the most frequent [2]. However, many cases of CAIS developing pure bilateral Sertoli cell adenoma have been previously described, but only a few with unilateral serous cystadenoma associated [3, 4].

In conclusion, the integration of clinical symptoms, physical examination, CT body scan and pathology studies is essential to confirm an accurate diagnosis of this uncommon syndrome.

The authors declare no conflict of interests.

\section{References}

1. Oakes MB, Eyvazzadeh AD, Quint E, et al. Complete androgen insensitivity syndrome - a review. J Pediatr Adolesc Gynecol 2008; 21: 305-10.

2. Manuel M, Katayama PK, Jones HW, Jr. The age of occurrence of gonadal tumors in intersex patients with a $\mathrm{Y}$ chromosome. Am J Obstet Gynecol 1976; 124: 293-300.

3. Baksu A, Kabukcuoglu F, Baksu B et al. Bilateral sertoli cell adenoma and serous cyst in a patient with androgen insensitivity syndrome. Eur J Obstet Gynecol Reprod Biol 2004; 114: 104-7.

4. Siminas S, Kokai G, Kenny SE. Complete androgen insensitivity syndrome associated with bilateral Sertoli cell adenomas and paratesticular leiomyomas: case report and review of the literature. J Pediatr Urol 2013; 9: e31-4.

\section{Address for correspondence}

Dr Iván Fernández-Vega $\mathrm{MD}, \mathrm{PhD}$

Service of Anatomic Pathology

Hospital Universitario de Araba-Txagorritxu,

C/Jose Achotegui s/n, E-01009,

Vitoria-Gasteiz, Alava, Spain

tel. 34945005107

e-mail: ivan_fernandez_vega@hotmail.com 